Meta

Journal des tradlucteurs

Translators' Journal

\title{
Les tables de salon
}

\section{Marisa Rondina et Rodrigue Gilbert}

Volume 22, numéro 2, juin 1977

URI : https://id.erudit.org/iderudit/003984ar

DOI : https://doi.org/10.7202/003984ar

Aller au sommaire du numéro

Éditeur(s)

Les Presses de l'Université de Montréal

ISSN

0026-0452 (imprimé)

1492-1421 (numérique)

Découvrir la revue

Citer cet article

Rondina, M. \& Gilbert, R. (1977). Les tables de salon. Meta, 22(2), 137-142.

https://doi.org/10.7202/003984ar

Ce document est protégé par la loi sur le droit d'auteur. L'utilisation des services d'Érudit (y compris la reproduction) est assujettie à sa politique d'utilisation que vous pouvez consulter en ligne.

https://apropos.erudit.org/fr/usagers/politique-dutilisation/ 


\section{LES TABLES DE SALON}

De cette étude, il ressort en premier lieu que le découpage de la réalité n'est pas du tout le même en français qu'en anglais. Analysons d'abord les notions de coffee table et de cocktail table.

The five terms coffee table, cocktail table, end table, lamp table et cigarette table refer to types of tables that are commonly found in living rooms. The coffee table is a "low table usually placed in front of a sofa to accommodate a coffee or other service, cups, plates, glasses and ashtrays ${ }^{1}{ }^{»}$. A cocktail table is a «low table for use in front of a sofa ${ }^{2} \gg$. Coffee table and cocktail table are synonyms; both the Webster's and the Random House define cocktail table as coffee table. Furthermore, the catalogue use these terms interchangeably.

Why then are there two terms? "The introduction of tea-coffee - , and chocolate drinking in the middle of the seventeenth century contributed to new table styles, just as cocktails have prompted the cocktail table and snacking the snack table... ${ }^{3} \gg$ "The coffee table gained in popularity as people began to move their after-dinner coffee out of the dining room ${ }^{4}$. The coffee table acquired its name because of the use to which it was put. In the same way, the term cocktail table was created. As the French say, «c'est une question de mode»!

1. Webster's Third New International Dictionary, Chicago, Encyclopedia Britannica Inc., 1966 , vol. 1 , p. 439.

2. Dorothy Stephat-De Van, Introduction to Home Furnishings, New York, Macmillan Co., 1971 , p. 275 .

3. The Practical Encyclopedia of Good Decorating and Home Improvement, New York,

Greystone Press, 1971, vol. 16, p. 3017.

4. House and Garden, février 1962, vol. 121, no 2, p. 134. 
Thus far, we have defined the terms coffee table and cocktail table. We have stated that they are synonyms. And we have also pointed out that the terms reflect the function of the object, and are specific, not generic, in nature.

Let us return to the question of synonymy. The use of the term cocktail table is only a recent phenomenon, and in addition, it is a catalogue phenomenon. In 1970, only coffee table was used; by 1972 both terms were found, and today, cocktail table has almost completely replaced coffee table.

The Simpsons-Sears catalogue writers are the only people who stray from this pattern; they make a distinction between a cocktail table, which is a long commode-type table, and a coffee table, which has four slender legs. Their usage, however, is not representative. We should therefore follow the general trend and consider coffee table and cocktail table as indeed being synonyms. Neither cost, size, nor style differentiate the two.

Coffee and cocktail tables can be rectangular, round or square. Smail square coffee tables that are placed in pairs are called either coffee tables or bunching tables. Many different materials are used for cocktail tables: wood, glass, chrome, rattan, etc. In a nutshell, coffee tables are found in a variety of styles and materials, and therefore, we can conclude that neither style nor material determine the type of table. The characteristics that do distinguish coffee tables from other tables are : 1) they are low tables (approx. $16^{\prime \prime}$ high) ; 2) they are usually placed in front of sofas ; 3) they hold a coffee or other service, etc.

Voyons maintenant les équivalents de coffee table et de cocktail table. Nous avons essayé, dans la majorité des cas, de baser notre acceptation ou notre refus des équivalents possibles sur des critères linguistiques en éliminant les questions de goût et de préférence personnelle.

Nous avons noté que dans la majorité des ouvrages consultés, «tables basses » et «tables de salon» sont les deux expressions qui ont été le plus souvent employées. Nous considérons qu'elles sont toutes deux parfaitement acceptables parce que premièrement elles correspondent à la démarche du français qui, comme nous l'avons déjà mentionné, a une plus forte tendance au générique que l'anglais. De plus, elles recoupent la même réalité que les termes anglais coffee table et cocktail table, et c'est peut-être là le critère le plus important, comme l'atteste l'exemple suivant : ces tables sont "agréables dans le coin salon [devant un divan ou un canapé], pour poser le plateau du café, les tasses ou les cendriers ${ }^{5}$ ».

Notons cependant que nous retrouvons ces deux expressions seulement dans les catalogues français ; en ce qui concerne les catalogues canadiens-français, nous retrouvons «table à café », «table à cocktail » et «table cocktail », ce qui est bien représentatif de la tendance locale qui donne des appellations spécifiques pour ces articles du mobilier.

Cependant, lorsque nous avons réellement besoin d'un terme spécifique, ce qui est le cas pour les catalogues ou dans des situations particulières, nous considérons que les trois expressions trouvées dans les catalogues canadiens sont de construc-

5. L'encyclopédie de la maîtresse de maison, Paris, Marabout, 1965, vol. 1, p. 232. 
tion acceptable parce qu'il existe déjà en français des modèles morphologiques semblables comme « service à thé 》, « service à café » et « service à liqueurs 》. De pius, l'expression tea table traduite par «table à thé » par Harrap est également acceptée. En ce qui concerne "table cocktail », nous croyons aussi que l'expression peut passer parce que le français d'aujourd'hui s'accommode bien de composés formés par juxtaposition.

Toutefois, d'autres équivalents pourraient être proposés. Il y a d'abord « tables auxiliaires », auxiliaire signifiant «qui aide par son concours (sans être indispensable) ${ }^{6} \gg$. L'expression nous paraît moins acceptable parce qu'elle est trop vague et qu'elle s'apparente trop à « table d'appoint » que nous discuterons plus tard.

Nous avons aussi «table de séjour » qui, construit sur le modèle de « table de salon », paraît acceptable en raison du parallélisme des expressions.

On pourrait aussi songer à «table buffet», ingénieux et acceptable, compte tenu du sens de buffet: "table où sont servis les pâtisseries, les rafraîchissements... ${ }^{3} \gg$

Il y aurait aussi des possibilités du côté de «table apéritif », qui nous semble acceptable parce que tout d'abord, l'apéritif décrit la même réalité que le cocktail : des personnes qui, avant le repas du soir, se réunissent autour d'une table pour discuter tout en "prenant un pot». En outre, ces mots jouissent d'une fréquence à peu près égale dans les deux cultures. Quant à sa structure, on peut de nouveau invoquer le fait que le français s'accommode de la juxtaposition comme dans le cas de «table cocktail 》 et «table buffet ». Enfin, notons que l'expression « table à apéritif » est également possible, mais que « table apéritif » présente l'avantage d'éviter le fâcheux hiatus.

Voilà, ce qui en gros, ressort de cette étude de coffee table et cocktail table ainsi que de leurs équivalents. Voyons maintenant les précisions qui s'imposent sur end, lamp et cigarette tables.

The end table, lamp table and cigarette table will now be considered as a group. The end table is a s small table placed beside a chair or at the end of a sofa to hold a lamp, ashtray, etc. ${ }^{8}$ \%. A lamp table is a table which is usually placed beside a chair or couch and on which a lamp is placed. "With the advent of oil lamps [...] and ultimately, electric lamps, the end table and lamp table became major ingredients of living room decoration. Sizes and styles are innumerable, and range from pedestals to campaign chest types, to lift-lid designs [...] to lean spindle-legged one-drawer types ${ }^{9}$. $\$$ A cigarette table is a "small, round table placed near a sofa or chair, on which an ashtray is placed. Cigarette tables are often pedestal tables, that is, tables with round or oval tabletops supported by a single base member, or guéridons, sinall round tables ${ }^{10} »$. Cigarette tables and

\footnotetext{
6. Paul Robert, Dictionnaire alphabétique et analogique de la langue française, Paris, Société du Nouveau Littré, Le Robert, 1966, t. 1, p. 124.

7. Ibid., p. 202

8. Martin Pegler, The Dictionary of Interior Design, New York, Crown Publishers, 1966,

p. 172

9. Martin Pegler, The Dictionary of Interior Design, p. 3018.

10. Martin Pegler, The Dictionary of Interior Design, p. 217.
} 
lamp tables, it is clear, are end tables. Of the three terms, end table is a generic term and is indicative of the table's position. On the other hand, cigarette table and lamp table are specific terms and indicate the use to which the table is put. We can compare the characteristics of these three end tables and contrast them to those of coffee and cocktail tables as follows :

\begin{tabular}{|c|c|c|c|c|}
\hline $\begin{array}{l}\text { TYPE OF } \\
\text { TABLE }\end{array}$ & $\begin{array}{c}\text { SIZE } \\
\text { (HEIGHT) }\end{array}$ & SHAPE & POSITION & $\begin{array}{c}\text { USE } \\
\text { (TABLE HOLDS) }\end{array}$ \\
\hline $\begin{array}{l}\text { 1. coffee/ } \\
\text { cocktail table }\end{array}$ & $\begin{array}{l}\text { low } \\
\left(16^{\prime \prime} \text { high }\right)\end{array}$ & $\begin{array}{l}\text { rectangular } \\
\text { square } \\
\text { round }\end{array}$ & $\begin{array}{l}\text { in front } \\
\text { of sofa }\end{array}$ & $\begin{array}{l}\text { service, plates, } \\
\text { glasses, etc. }\end{array}$ \\
\hline $\begin{array}{l}\text { 2. end table } \\
\text { (G) }\end{array}$ & $\begin{array}{l}\text { low, but } \\
\text { higher than } 1 . \\
\left(20^{\prime \prime} \text { high }\right)\end{array}$ & $\begin{array}{l}\text { rectangular } \\
\text { square } \\
\text { round * } \\
\text { (smaller than 1.) }\end{array}$ & $\begin{array}{l}\text { beside } \\
\text { sofa, } \\
\text { chair }\end{array}$ & lamp, ashtray \\
\hline 3. lamp table & $20^{\prime \prime}$ high & same as 2 & $\begin{array}{l}\text { usually } \\
\text { beside sofa, } \\
\text { chair }\end{array}$ & lamp \\
\hline $\begin{array}{l}\text { 4. cigarette } \\
\text { table }\end{array}$ & $17^{\prime \prime}$ high & $\begin{array}{l}\text { round } \\
\text { (smaller than 2.) }\end{array}$ & $\begin{array}{l}\text { beside sofa, } \\
\text { chair }\end{array}$ & ashtray \\
\hline
\end{tabular}

Let us say a few words about nests of tables. These tables, also known as nesting tables, are tables that telescope into each other. L'équivalent français est «tables gigognes» et ne pose aucune difficulté. Quant aux bunching tables, on retrouve comme traduction « tables basses » et «tables juxtaposables 》.

Pour traduire end table, nous avons une fois de plus «table basse », terme générique utilisé dans les catalogues français et qui paraît encore ici acceptable : «... un grand canapé en cuir brun s'étale, flanqué par des tables basses où sont posées de belles urnes montées en lampes ${ }^{11}$ ».

On retrouve aussi l'expression "petites tables » qui pourrait être utile s'il faut distinguer les "tables basses " du type coffee table de celles du type end table.

Dans les catalogues canadiens, on trouve «table de bout», calque évident de la forme anglaise, et qu'on aurait profit à remplacer par l'un des équivalents suivants : 1) «Table d'appoint»: acceptable parce que le complément convient bien à la fonction de ce type de table. On peut aussi donner comme synonyme «tables de complément » et «table d'appui » d'autant plus que appui signifie « au propre et au figuré ce sur quoi un objet repose (sans que cela fasse partie de l'objet) ${ }^{12} \gg$;2) «Petite table de salon » ou «petite table de séjour »: acceptable pour les raisons données plus haut. Quant à «table d'extrémité », cette expression paraît être, comme table de bout », un calque de l'anglais et d'un caractère moins « orthodoxe».

11. 1000 idées de décoration, Paris, * Grands dictionnaires de la maison », Denoël, 1974, p. 97.

12. Henri Benac, Dictionnaire des synonymes, Paris, Librairie Hachette, 1956, p. 53. 
Maintenant, voyons ce que nous avons recueilli pour lamp table. Dans les catalogues français, on retrouve «table basse », acceptable toujours pour les mêmes raisons ; on retrouve aussi " table basse éclairante » et «table luminaire », moins acceptables parce qu'on a l'impression que ce sont les tables qui éclairent et non la lampe, mais cependant, la langue n'est pas toujours aussi rigoureuse. Par contre, «table luminaire », du point de vue de la structure, est tout aussi acceptable que « table apéritif».

Dans les catalogues canadiens, on trouve : 1) «Table à lampe», avec le complément de destination comme dans «table à café »;2) "Table de lampe », la préposition «de » conviendrait peut-être, avec un complément d'action ; 3) « Table d'éclairage » et «petite table d'éclairage »: l'un et l'autre ont l'avantage d'être très précis.

Résumons donc notre étude par le tableau suivant:

\begin{tabular}{|c|c|c|c|c|}
\hline $\begin{array}{l}\text { TERMES } \\
\text { ANGLAIS }\end{array}$ & SYNONYMES & $\begin{array}{l}\text { TERMES } \\
\text { FRANÇAIS }\end{array}$ & $\begin{array}{l}\text { TERMES } \\
\text { FRANÇAIS- } \\
\text { CANADIENS }\end{array}$ & $\begin{array}{l}\text { TERMES } \\
\text { PROPOSÉS }\end{array}$ \\
\hline coffee table (S) & cocktail table (S) & $\begin{array}{l}\text { table basse }(G) \\
\text { table de salon }(G) \\
\text { table salon }(G) *\end{array}$ & $\begin{array}{l}\text { table à café (S) } \\
\text { table à cocktail } \\
\text { (S) } \\
\text { table cocktail (S) }\end{array}$ & $\begin{array}{l}\text { table auxiliaire } \\
(G) * \\
\text { table de séjour (G) } \\
\text { table buffet (S) } \\
\text { table apéritifs (S) }\end{array}$ \\
\hline coffee table (S) & $\begin{array}{l}\text { bunching table } \\
\text { (S) }\end{array}$ & $\begin{array}{l}\text { table basse }(\mathrm{G}) \\
\text { table } \\
\text { juxtaposable }(\mathrm{S})\end{array}$ & $\begin{array}{l}\text { table } \\
\text { juxtaposable }\end{array}$ & \\
\hline cocktail table & $\begin{array}{l}\text { voir: } \\
\text { coffee table }\end{array}$ & & & \\
\hline end table (S) * & & $\begin{array}{l}\text { table basse (G) } \\
\text { petite table (G) }\end{array}$ & $\begin{array}{l}\text { table de bout } \\
\text { (S) }\end{array}$ & $\begin{array}{l}\text { table d'appoint (G) } \\
\text { table de } \\
\text { complément }(G) \\
\text { table d'appui }(G) \\
\text { petite table de } \\
\text { salon (S) } \\
\text { petite table de } \\
\text { séjour (S) } \\
\text { table d'extrémité } \\
(\mathrm{S}) *\end{array}$ \\
\hline lamp table (S) & & $\begin{array}{l}\text { table basse (G) } \\
\text { table basse } \\
\text { éclairante (S) } \\
\text { table-luminaire } \\
(\mathrm{S})\end{array}$ & $\begin{array}{l}\text { table à lampe (S) } \\
\text { table de lampe } \\
(\mathrm{S}) *\end{array}$ & $\begin{array}{l}\text { table d'éclairage } \\
\text { (S) } \\
\text { petite table } \\
\text { d'eclairage (S) }\end{array}$ \\
\hline cigarette table (S) & $\begin{array}{l}\text { pedestal table } \\
\text { (G) } \\
\text { guéridon (S) }\end{array}$ & $\begin{array}{l}\text { table basse }(G) \\
\text { petite table }(G) \\
\text { guéridon }(G)\end{array}$ & $\begin{array}{l}\text { table à cigarettes } \\
(\mathbf{S}) *\end{array}$ & $\begin{array}{l}\text { table fumeurs (S) } \\
\text { table fumoir (S) } \\
\text { table tabac (S) } \\
\text { table à cendriers } \\
\text { (S) } \\
\text { table à fumer (S) * }\end{array}$ \\
\hline nest of tables & nesting table & tables gigognes & tables gigognes & \\
\hline
\end{tabular}

* Termes rejetés. 
En ce qui concerne cigarette table maintenant, les catalogues français parlent de "petite table » et de "table basse », ce qui atteste bien le caractère générique de ces expressions. On parle aussi de "guéridon » qui, dans ce cas, paraît adéquat, les guéridons étant "de toutes les tailles, avec un plateau rond [...] ils ont soit un pied central, soit trois pieds ${ }^{13} \gg$. D'ailleurs, les illustrations de ces catalogues corroborent cette définition. Les catalogues canadiens, eux, donnent « table à cigarettes ». Encore ici, le calque n'est pas loin et nous incite à la prudence.

Dans la même famille synonymique, nous trouvons encore «table fumeur », «table fumoir» et «table tabac », avec le même modèle structural que « table apéritif »; "table à cendriers», avec complément de destination; «table à fumer », sur le modèle de table à repasser.

MARISA RONDINA RODRIGUe GiLberT

13. La Décoration de $A$ à $Z$, Mille renseignements, Paris, * Grands dictionnaires de la maison », Denoël, 1974, p. 518. 